\title{
Response-response compatibility during bimanual movements: Evidence for the conceptual coding of action
}

\author{
ELIOT HAZELTINE \\ University of Iowa, Iowa City, Iowa
}

\begin{abstract}
The present study investigated response-response (R-R) compatibility in a bimanual keypressing task. Numeric and spatial stimuli were used to cue responses for each hand. Two groups of participants differed in terms of the stimulus-response mappings for the numeric stimuli. For one group, the numeric stimuli were mapped so that the same number for each hand indicated responses that were anatomically compatible (e.g., index finger of both hands). For the other group, the same number for each hand indicated responses that were left-right compatible (e.g., leftmost finger of both hands). The spatial stimuli were mapped in a spatially compatible manner to the responses for both groups. For numeric stimuli, reaction times (RTs) were faster when the same number indicated the response for each hand, regardless of the mapping. For the spatial stimuli, RTs were determined not only by the pairing of stimuli or responses, but also by how the responses were indicated by numeric stimuli. The results indicate that $\mathrm{R}-\mathrm{R}$ compatibility effects are mediated by abstract codes that reflect individuals' conceptualizations of their actions.
\end{abstract}

Human performance in choice reaction time tasks depends on the pairings of stimuli and responses. Such effects, termed stimulus-response ( $\mathrm{S}-\mathrm{R})$ compatibility effects, are widely studied because they reflect the processing architecture that transforms stimuli into the appropriate responses - a fundamental cognitive operation (see, e.g., Kornblum, Hasbroucq, \& Osman, 1990). Of course, the actual stimuli or responses do not produce compatibility effects; their representations do. This point is driven home by studies that demonstrate the importance of environmental feedback in determining compatibility effects. For example, Hommel (1993) showed that compatibility effects between the location of the critical stimulus and location of the response (i.e., the Simon effect) could be reversed by associating task-relevant environmental consequences with the responses (see also Guiard, 1983).

Less widely studied are response-response (R-R) compatibility effects. Such effects can be observed when individuals simultaneously produce two responses-one with each hand-to two stimuli. Under such conditions, reaction times (RTs) are faster when corresponding responses are made with the two hands (Heuer, 1995). For example, Lien and Proctor (2000) performed a dual-task experiment

This work was supported by the Aviation Operations Systems Program at NASA Ames Research Center. Herbert Heuer, Rich Ivry, Mei-Ching Lien, Gordon Logan, and Robert Proctor provided extremely helpful comments on a draft of this manuscript. The author is also grateful to Michelle Devins, Ali Mattu, and Paul Aparicio for their assistance in collecting the data. Correspondence concerning this article should be addressed to E. Hazeltine, Department of Psychology, University of Iowa, 11 Seashore Hall E, Iowa City, IA 52242 (e-mail: eliot-hazeltine@ uiowa.edu). in which participants performed two keypressing tasks, and the stimuli for the two tasks were separated by a brief, varying interval. RT benefits were observed when the relative spatial locations of the keypresses of the two tasks matched as opposed to when they did not. Because the stimuli for the two tasks were unrelated, the effect was attributed to correspondence effects between spatial codes associated with the two responses.

For the most part, the explanations of R-R compatibility present a dichotomy between stimulus-based accounts that emphasize crosstalk between signals relating to sensory feedback and response-based accounts that emphasize crosstalk between spatial or anatomical properties of responses. Stimulus-based accounts propose that R-R compatibility is perceptual: Compatible responses yield sensory consequences that can be efficiently coded (see, e.g., Mechsner, Kerzel, Knoblich, \& Prinz, 2001). Responsebased accounts propose that R-R compatibility results from crosstalk between motor programming processes (e.g., Heuer, 1995).

However, a recent study by Lien, Schweickert, and Proctor (2003) has suggested a third account of R-R compatibility. These researchers used a dual-task procedure in which stimuli for two tasks, one performed by the left hand and the other performed by the right hand, were presented in quick succession. The two stimuli could belong to the same task (e.g., even-odd categorization of a number) or different tasks (e.g., even-odd categorization and vowel-consonant categorization). The R-R compatibility effects were restricted largely to trials in which the two categories were the same (e.g., vowel and vowel). Thus, the researchers concluded that the locus of R-R compatibility effects is in response selection processes. 
The present study explores the nature of the representations that underlie R-R compatibility effects. Specifically, do the interactions between simultaneously produced responses involve stimulus, response, or abstract representations? In contrast to previous studies, the abstract representations examined here are flexible in that they are not strictly contingent on the particular stimuli or responses present on a given trial. Instead, they depend on the participants' conceptualization of the task. Therefore, the same pairs of stimuli and responses may in one case be compatible and in another case be incompatible, because the pairs invoke different or similar codes during $\mathrm{S}-\mathrm{R}$ translation.

In a test of these accounts, participants performed a keypressing task in which numeric and spatial stimuli were used to indicate responses made by the two hands. Stimuli on the right half of the display indicated keypresses to be made with the right hand, and stimuli on the left half of the display indicated keypresses to be made with the left hand. There were two types of trials: unimanual trials, in which only a left-hand or right-hand stimulus appeared, and bimanual trials, in which both left-hand and right-hand stimuli appeared simultaneously. On bimanual trials, participants were instructed to produce keypresses with both hands simultaneously.

The numeric stimuli were used to manipulate the way that the participants conceptualized their responses (Figure 1). For participants in the anatomical group, the numbers indicated which finger should press a key (e.g., index finger). For participants in the left-right group, the numbers indicated which key, numbered from left to right, should be pressed. The spatial stimuli were used to test whether the manner in which the responses were

\section{Left-right: $\quad 1234 \quad 1234$}

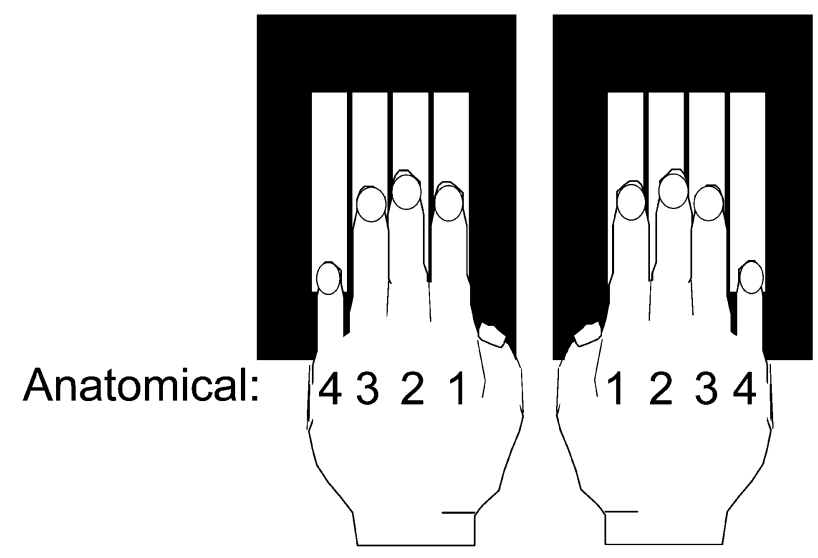

Figure 1. The $\mathbf{S}-\mathbf{R}$ associations for the number stimuli for the two experimental groups. For the left-right group (top row), numbers indicate the keypress from the leftmost (1) to the rightmost (4) location for each hand. For the anatomical group (bottom row), the numbers indicate the appropriate keypress in a mirrorsymmetric fashion corresponding to the digits, so that " 1 " designates the innermost keypress and "4" designates the outermost keypress. conceptualized affected R-R compatibility. For these stimuli, the same compatible $\mathrm{S}-\mathrm{R}$ mappings were used for all participants. Any combination of the two stimulus types was possible on a given trial.

As illustrated in Figure 2, on bimanual trials, the relationship between the keypresses of the two hands can be categorized as either anatomically compatible, left-right compatible, or incompatible. Anatomically compatible responses are those in which the same finger produced the keypress for the left and right hands-for example, the index finger of both hands. Left-right compatible responses are those in which the two keypresses shared the same relationship to the keyboards in allocentric spacefor example, the leftmost key of both response boards. Responses fulfilling neither of these criteria were considered incompatible.

The principal question is whether the benefits of anatomical compatibility and left-right compatibility afforded the spatial stimuli will depend on the S-R mapping for the numeric stimuli. If R-R compatibility is based on the similarity between the relative spatial locations of the keypresses or motor outputs for the two hands, then it should be the same regardless of the S-R mappings for the numeric stimuli. Similarly, if the spatial stimuli directly access representations of spatially compatible responses, then the mapping for the number stimuli should not affect R-R compatibility when spatial stimuli are used. However, if R-R compatibility is based on interactions between abstract codes, then different patterns of RTs should be observed for the two types of mappings. That is, when the numbers encourage an anatomical or mirrorsymmetric coding of the responses, spatial stimuli should produce anatomical compatibility effects, and when the numbers encourage left-right coding of the responses, spatial stimuli should produce left-right compatibility effects.

In sum, if compatibility effects reflect interactions between abstract codes rather than interactions between stimulus or response representations, then RTs for the spatial stimuli should depend on the conceptualization of the responses. Although the $\mathrm{S}-\mathrm{R}$ associations for the spatial stimuli are the same for both mapping groups, the conceptualization of the responses is manipulated with the numeric stimuli. To the best of my knowledge, this experiment provides the first test of whether R-R compatibility effects are affected by the way individuals conceptualize their actions independent of the spatial and motoric aspects of the responses.

\section{METHOD}

\section{Participants}

Thirty-four community college students (14 male) between the ages of 17 and 25 were paid to participate. Data from 2 participants were eliminated from the analyses because of high error rates.

\section{Apparatus and Stimuli}

The experiment was implemented using E-Prime software on PC 586 computers. The stimuli were white presented against a black background. A fixation cross subtending $0.5^{\circ}$ of visual angle remained present throughout each block. On bimanual trials, stimuli 


\section{Anatomical Mapping Left-Right Mapping}

\begin{tabular}{|c|c|c|c|c|}
\hline $\mathrm{N}-\mathrm{N}$ & $\begin{array}{c}\text { Left } \\
\text { Stimulus }\end{array}$ & $\begin{array}{c}\text { Right } \\
\text { Stimulus }\end{array}$ & $\begin{array}{c}\text { Left } \\
\text { Stimulus }\end{array}$ & $\begin{array}{c}\text { Right } \\
\text { Stimulus }\end{array}$ \\
\hline $\begin{array}{l}\text { Anatomically } \\
\text { Compatible }\end{array}$ & 2 & 2 & 3 & 2 \\
\hline $\begin{array}{l}\text { Left-Right } \\
\text { Compatible }\end{array}$ & 3 & 2 & 2 & 2 \\
\hline Incompatible & 4 & 2 & 1 & 2 \\
\hline \multicolumn{5}{|l|}{$\mathrm{N}-\mathrm{S}$} \\
\hline $\begin{array}{l}\text { Anatomically } \\
\text { Compatible }\end{array}$ & 2 & $-*--$ & 3 & $-*--$ \\
\hline $\begin{array}{l}\text { Left-Right } \\
\text { Compatible }\end{array}$ & 3 & $-*--$ & 2 & $-*--$ \\
\hline Incompatible & 4 & $-*--$ & 1 & $-*--$ \\
\hline \multicolumn{5}{|l|}{ S-N } \\
\hline $\begin{array}{l}\text { Anatomically } \\
\text { Compatible }\end{array}$ & $--*$ & 2 & $--*$ & 2 \\
\hline $\begin{array}{l}\text { Left-Right } \\
\text { Compatible }\end{array}$ & $-*--$ & 2 & $-*--$ & 2 \\
\hline Incompatible & *-- - & 2 & *-- - & 2 \\
\hline \multicolumn{5}{|l|}{ S-S } \\
\hline $\begin{array}{l}\text { Anatomically } \\
\text { Compatible }\end{array}$ & $--*$ & $-*--$ & $--*$ & $-*--$ \\
\hline $\begin{array}{l}\text { Left-Right } \\
\text { Compatible }\end{array}$ & $-*-$ & $-*-$ & $-*-$ & $-*-$ \\
\hline Incompatible & *-- - & $-*--$ & $*---$ & $-*--$ \\
\hline
\end{tabular}

Figure 2. The stimuli and responses for the two mapping groups. The rows labeled $\mathrm{N}-\mathrm{N}$ show sample stimuli for number-number trials; the $\mathrm{N}-\mathrm{S}$ rows show sample stimuli for number-spatial trials; $\mathrm{S}-\mathrm{N}$ rows show sample stimuli for spatial-number trials; and S-S rows, sample stimuli for spatial-spatial trials. In each case, anatomically compatible responses involve keypresses with the two middle fingers; left-right compatible responses involve keypresses with the left ring finger and right middle finger; and incompatible responses involve keypresses with left pinky finger and right middle finger.

were presented simultaneously $4.0^{\circ}$ to the left and right of fixation. On unimanual trials, a stimulus was presented only on the side corresponding to the moving hand.

Left-hand responses were made using the keys a, s, $\mathrm{d}$, and $\mathrm{f}$. Right-hand responses were made using the keys $h, j, k$, and 1 . The numeric stimuli were the numbers $1-4$. The spatial stimuli were $*_{---}$, -*--, --*-, and ---*, indicating the four possible responses on each hand in a spatially compatible fashion (see Figure 2). Each finger on a given hand was associated with one anatomically compatible combination, one left-right compatible combination, and two incompatible combinations. Thus, comparisons between conditions were completely balanced in terms of the particular fingers comprising the responses.

\section{Procedure}

Participants were told the S-R mapping and instructed to respond to both stimuli at the same time as quickly and accurately as possible. The participants were assigned to one of two groups that differed according to the S-R mapping for the numbers (Figure 1). For the left-right mapping group, the numbers on both sides of fix- ation indicated the appropriate keypress in a left-right fashion, so that " 1 " indicated the leftmost key; " 2 ," the key second from the left; " 3 ," the key third from the left; and "4," the rightmost key. For the anatomical mapping group, the numbers indicated the appropriate keypress in a mirror fashion, so that 1 indicated the key under the index finger; " 2 ," the key under the middle finger; and the " 3 ," under the ring finger; and " 4 ," the key under the pinky finger. Note that these two mappings are identical for the right hand. The assignments for the spatial stimuli were the same for both groups.

Participants completed eight experimental blocks, each consisting of 96 trials. Only number stimuli were used during the first two blocks. For these blocks, the 96 trials consisted of four instances of each possible combination of left-hand stimulus and right-hand stimulus ( 4 left-hand stimuli $\times 4$ right-hand stimuli $\times 4$ instances $=64$ trials $)$ plus four instances of unimanual trials in which each left- and righthand stimulus was paired with no stimulus on the other hand (4 stimuli $\times 2$ hands $\times 4$ instances $=32$ trials).

After the participants completed the first two blocks, the experimenter informed the participants that spatial stimuli would appear 
on some trials and explained the $\mathrm{S}-\mathrm{R}$ mapping for these stimuli. The explanation was identical for both mapping groups. On the remaining six blocks, the 96 trials consisted of one instance of all possible combinations of left- and right-hand stimuli (8 left-hand stimuli $\times 8$ right-hand stimuli $=64$ trials) plus two instances of trials in which only a single stimulus appeared on either the left or right ( 8 stimuli $\times 2$ hands $\times 2$ instances $=32$ trials $)$. The order of the stimuli was randomized by the E-Prime software.

Each trial began with the presentation of a fixation cross for $1 \mathrm{sec}$. The stimuli were then presented and remained on the screen for $2 \mathrm{sec}$. The stimuli were then extinguished, and only the fixation cross remained visible for a 1 -sec interval. The next trial began immediately after the completion of this poststimulus interval. Thus, $4 \mathrm{sec}$ elapsed between consecutive onsets of the stimuli, and responses were recorded at any point within $3 \mathrm{sec}$ after the onset.

\section{RESULTS}

Analysis was restricted to the final four sessions of the experiment. For bimanual trials, RTs were analyzed only if both keypresses were correct $(90 \%)$ and produced within 150 msec of each other (98\%). RTs were averaged across the two hands to produce a single RT for these trials. The mean RTs across trial types for the four stimulus combinations are shown in Figure 3.

\section{Conceptual Codes}

The critical question posed by the experiment was whether the two mapping groups would show different patterns of compatibility effects on the spatial-spatial trials (Figure 3, panel labeled S-S). In short, would R-R compatibility interact with mapping group, even though the two groups used identical S-R mappings for the spatial stimuli? The RTs from spatial-spatial trials were submitted to a two-way ANOVA, with mapping (anatomical or left-right) as a between-subjects factor and trial type (anatomically compatible, left-right compatible, or incompatible) as a within-subjects factor.

Both main effects were reliable: RTs were shorter for the anatomical group than for the left-right group (956 vs. $1,084 \mathrm{msec}$, respectively) with spatial-spatial stimuli $[F(1,30)=13.72, p<.001]$, and incompatible trials $(1,260 \mathrm{msec})$ were performed more slowly than anatomically compatible $(1,054 \mathrm{msec})$ and left-right compatible trials $(1,054 \mathrm{msec})[F(2,60)=89.77, p<.0001]$. Most important, the interaction between group and trial type was also reliable $[F(2,60)=36.36, p<.0001]$, indicating that anatomically compatible responses were produced fastest for the anatomical mapping group, whereas left-right compatible trials were produced fastest for the left-right mapping group.

The interaction for the spatial-spatial trials reflects a $300-\mathrm{msec}$ reversal in the direction of the advantage for left-right and anatomically compatible trials depending on the mapping for the numeric stimuli, which were not present on these trials. The compatibility effects, computed by subtracting the RTs for the corresponding trial type from the RTs for the incompatible trials, indicate
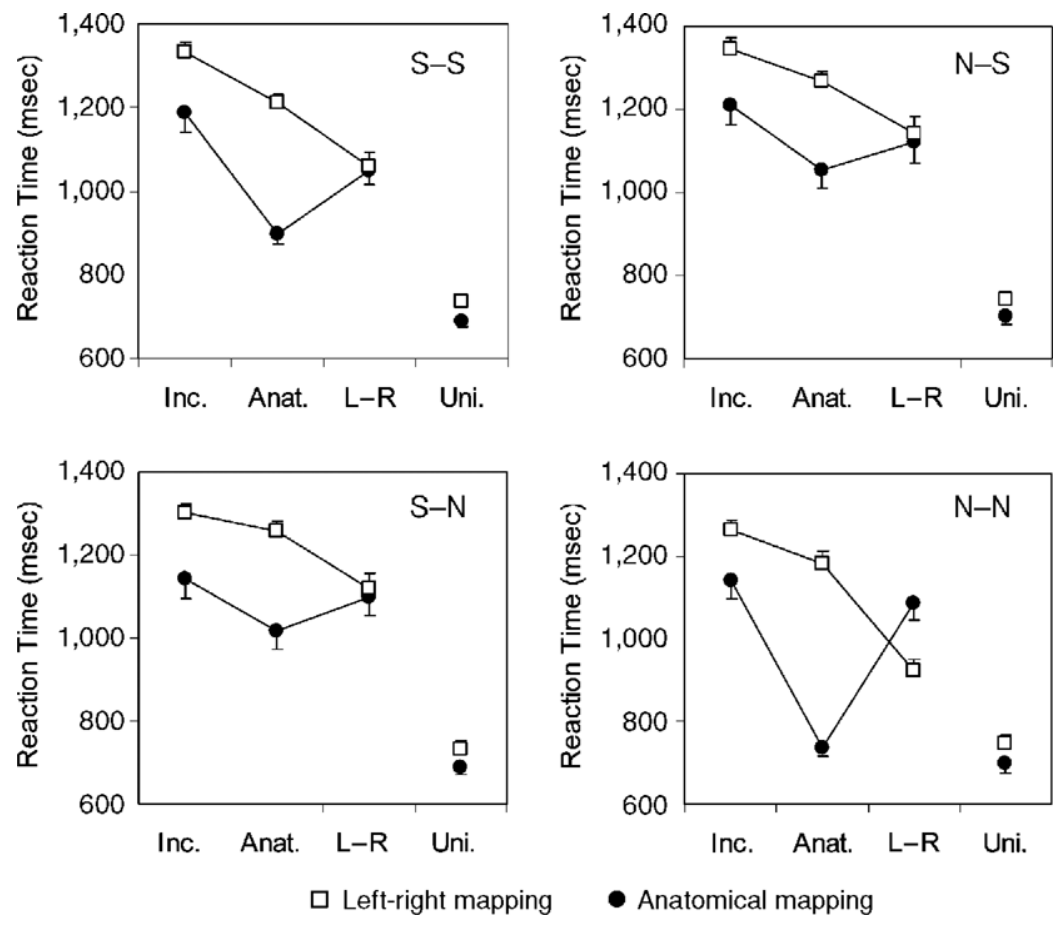

Figure 3. Reaction times for the four trial types for the two mapping groups. Open squares indicate scores for the left-right mapping group; filled circles indicate compatibility scores for the anatomical mapping group. Error bars represent standard error of the mean. Inc., incompatible trial; Anat., anatomically compatible trial; $\mathbf{L}-\mathbf{R}$, left-right compatible trial; Uni., unimanual trial. 
that the mapping for the number stimuli played a major role in the R-R compatibility of the spatial stimuli (Figure 4). This pattern held despite the fact that the spatialspatial stimuli were mapped to the same responses, and the instructions for the spatial cues were identical for the two mapping groups. Thus, the benefit afforded particular combinations of responses is not driven by either the stimuli or the responses of the particular trial. Instead, the mapping for the number stimuli determined the conceptual coding of the responses: When the mapping of the numbers encouraged individuals to conceptualize their actions as keypresses with particular fingers or along an axis mirror-symmetric to the body midline, anatomically compatible responses were produced fastest. When the mapping of the numbers encouraged individuals to conceptualize their actions as keypresses along an allocentric axis, left-right compatible responses were produced fastest.

Although the interaction reflected a clear asymmetry in the magnitude of the compatibility effects, the anatomical group performed the left-right compatible trials $138 \mathrm{msec}$ faster than the incompatible trials $[t(15)=$ $6.33, p<.0001]$, and the left-right group performed the anatomically compatible trials $122 \mathrm{msec}$ faster than the incompatible trials $[t(15)=5.76, p<.0001]$. These effects may reflect codes that are not purely based on a left-right or anatomical conceptualization of the responses. Instead, both anatomical and left-right aspects of responses may be included in their representation for both groups, but the mapping of the number stimuli determines which of these aspects are more salient.

Performance on each of the other three possible stimulus combinations (number-number, number-spatial, and spatial-number) showed a similar pattern. For the RTs, there were main effects of mapping [number-number, $F(1,30)=10.05, p<.005$; number-spatial, $F(1,30)=$ $5.50, p<.05$; spatial-number, $F(1,30)=7.78, p<.01]$ and trial type [number-number, $F(2,60)=117.43, p<$
.0001 ; number-spatial, $F(2,60)=39.14, p<.0001$; spatial-number, $F(2,60)=29.92, p<.0001]$. In these cases, the anatomical group performed faster than the left-right group, and incompatible trials were performed more slowly than the left-right or anatomically compatible trials (see Figure 3). The interaction between mapping and trial type was also significant for each of the other stimulus combinations [number-number, $F(2,60)=$ $163.16, p<.0001$; number-spatial, $F(2,60)=15.03$, $p<.0001$; spatial-number, $F(2,60)=27.01, p<.0001]$, reflecting the same advantage for anatomically compatible responses for the anatomical mapping and left-right compatible responses for the left-right mapping, although the magnitude of the interaction varied considerably.

The magnitude of the mapping $\times$ trial type interaction was largest for the number-number stimuli, which produced a 600-msec reversal in the difference between the anatomical and left-right compatible trials across the two mapping groups. In this case, the interaction was partly attributable to stimulus repetition effects: The simultaneous presentation of the same number indicated anatomically compatible responses for the anatomical mapping group and left-right compatible responses for left-right mapping group. However, the stimulus repetition account cannot explain the interaction for trials in which one response is cued spatially and the other numerically. It also cannot explain the interaction for trials in which both responses are cued spatially, because the S-R mappings are the same on these trials for the anatomical and left-right mapping groups.

\section{Dual-Task Costs}

Dual-task costs were computed by subtracting the unimanual RTs from the RTs on the bimanual, incompatible trials. Dual-task costs averaged $524 \mathrm{msec}$. The magnitude of the costs were large given that the mean unimanual RT was $670 \mathrm{msec}$, likely reflecting interactions between two

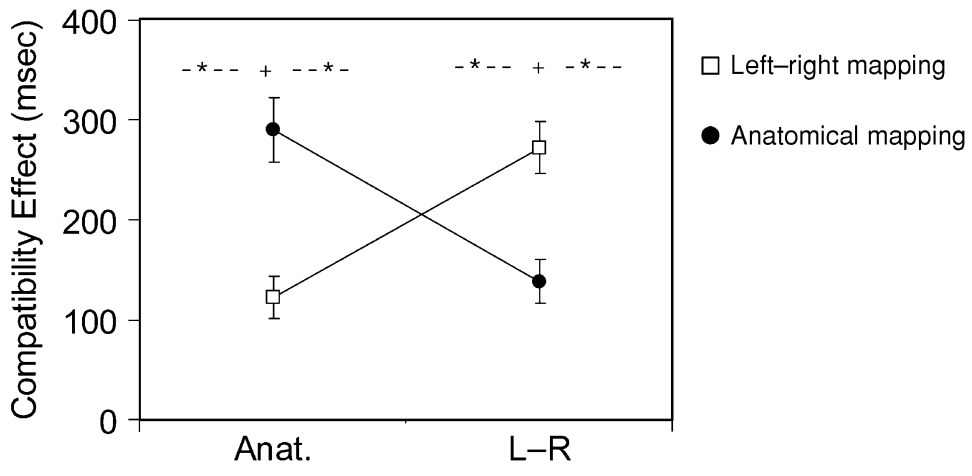

Figure 4. The magnitude of compatibility effects, calculated by subtracting the RTs on either anatomically (Anat.) or left-right $(L-R)$ compatible trials from the RTs on incompatible trials, for trials with spatial-spatial stimuli. Example stimuli are shown above the corresponding data points. Open squares indicate the scores for the left-right mapping group; filled circles indicate the compatibility scores for the anatomical mapping group. Error bars represent standard error of the mean. 
four-choice tasks, both with manual responses. The costs were submitted to a three-way ANOVA with hand, stimulus combination, and mapping as factors. The factor hand indicated both the side of the stimulus and the side of the response. There were significant effects of hand $[F(1,30)=$ $15.64, p<.0001]$, stimulus combination $[F(3,90)=$ $17.21, p<.0001]$, and mapping $[F(1,30)=5.99, p<.05]$, but no reliable interactions. The effect of hand (left: $507 \mathrm{msec}$; right: $540 \mathrm{msec}$ ) resulted from the fact that on unimanual trials, right-hand responses were performed more quickly than left-hand responses, but on bimanual incompatible trials, participants were instructed to make both responses simultaneously, and no difference was observed. The effect of stimulus combination (numbernumber, $445 \mathrm{msec}$; number-spatial, $505 \mathrm{msec}$; spatialnumber, $452 \mathrm{msec}$; spatial-spatial, $501 \mathrm{msec}$ ) was consistent with an advantage for trials in which number stimuli were presented in the right visual field and therefore processed by the left hemisphere. The effect of mapping (anatomical, $476 \mathrm{msec}$; left-right, $571 \mathrm{msec}$ ) indicated smaller mean costs for the anatomical group. Note that, because the analysis is based on dual-task costs over and beyond unimanual RTs, these effects must result from interactions between simultaneously produced responses.

\section{Accuracy}

ANOVAs mirroring those performed on the RT data were performed on the proportions of correct responses. These analyses generally produced the same patterns of results, with conditions producing faster RTs also producing higher proportions of correct responses. There were no cases in which findings in the RT data appeared to result from a speed-accuracy trade-off.

\section{DISCUSSION}

The present findings indicate that compatibility effects between simultaneously produced responses result, at least in part, from interactions between the conceptualizations of the actions. Even spatially compatible stimuli, which, according to some accounts, directly activate the corresponding responses (e.g., Kornblum et al., 1990), produce $\mathrm{R}-\mathrm{R}$ compatibility effects that are mediated by the context of the experimental setting. These effects are of the order of hundreds of milliseconds, indicating that the conceptualization of the action plays a major role in response selection.

Fitts and Seeger (1953) demonstrated that the compatibility of an ensemble of S-R pairs was dependent on the relationships between all members of the stimuli and all members of the responses, a phenomenon termed set-level compatibility (see also Kornblum et al., 1990; Proctor \& Reeve, 1990; Teichner \& Krebs, 1974). Given that the effect of mapping on unimanual trials was small (about $40 \mathrm{msec}$ ) compared with the effect of R-R compatibility, this form of compatibility does not seem to play a major role in the pattern of present results. Nonetheless, because the compatibility effects for the spatial stimuli depend on the mappings for the number stimuli, these findings might be viewed as an extension of set-level compatibility.

However, whereas set-level compatibility relates to $\mathrm{S}-\mathrm{R}$ mappings as a whole, it does not address interactions between specific $\mathrm{S}-\mathrm{R}$ rules linked to individual responses. The R-R compatibility effects cannot be treated as an instance of set-level compatibility unless it is assumed that the two keypresses on bimanual trials are represented as a single response and that the number and spatial stimuli form a single task set. The novel contribution of these findings is that, by evaluating R-R compatibility between concurrently produced responses, it is possible to detect differences in the $\mathrm{S}-\mathrm{R}$ associations for two sets of identical, spatially compatible mappings.

The conceptual compatibility effect is likely related to category repetition effects: When multiple stimuli are associated with a particular response category, repetitions of the category, even when the particular stimuli differ, produce RT benefits (Campbell \& Proctor, 1993; Logan \& Schulkind, 2000; Pashler \& Baylis, 1991). Lien, Schweickert, and Proctor (2003) showed that R-R compatibility effects were largely confined to within task set category repetitions, especially when the task sets for the two hands could be predicted. For these reasons, the researchers concluded that R-R compatibility stemmed from central processes, consistent with the present study.

Critically, the present case differs from these experiments in that there is no explicit category linking the responses for the two hands during trials with spatial stimuli. Moreover, unlike the results of Lien, Schweickert, and Proctor (2003), the effects of the mapping for the number stimuli were pronounced even for the spatialspatial trials in which no number stimuli appeared. That is, the spatially cued responses were not freed of their associations established by the number stimuli, and these associations produced powerful R-R compatibility effects.

Mechsner, Kerzel, Knoblich, and Prinz (2001) manipulated the sensory feedback associated with repetitive motor actions and found that R-R compatibility effects were determined by spatial correspondence between the feedback stimuli associated with the responses of the two hands rather than correspondence between the underlying movements. How important are sensory feedback and environmental consequences in governing compatibility effects? The present results suggest that, although environmental consequences may provide a powerful means of manipulating the conceptualization of the response, the critical determinants of compatibility effects may be somewhat independent of concurrent or expected sensory events. Bimanual responses in the present experiment interacted with each other based not on stimulus or response information-these were identical for the two mapping groups on the spatial-spatial trials-but according to how the responses were internally coded. $\mathrm{R}-\mathrm{R}$ compatibility does not depend on the correspondence between perceptual or spatial codes; it stems from the correspondence between $\mathrm{S}-\mathrm{R}$ rules. 


\section{REFERENCES}

Campbell, K. C., \& Proctor, R. W. (1993). Repetition effects with categorizable stimulus and response sets. Journal of Experimental Psychology: Learning, Memory, \& Cognition, 19, 1345-1362.

FitTs, P. M., \& SEEgER, C. M. (1953). S-R compatibility: Spatial characteristics of stimulus and response codes. Journal of Experimental Psychology, 46, 199-210.

GUIARD, Y. (1983). The lateral coding of rotations: A study of the Simon effect with wheel-rotation responses. Journal of Motor Behavior, 15, 331-342.

HEUER, H. (1995). Models for response-response compatibility: The effects of the relation between responses in a choice task. Acta Psychologica, 90, 315-332.

Hommel, B. (1993). Inverting the Simon effect by intention: Determinants of direction and extent effects of irrelevant spatial information. Psychological Research, 55, 270-279.

Kornblum, S., HasbroucQ, T., \& Osman, A. (1990). Dimensional overlap: Cognitive basis for stimulus-response compatibility: A model and taxonomy. Psychological Review, 97, 253-270.

Lien, M.-C., \& Proctor, R. W. (2000). Multiple spatial correspondence effects on dual-task performance. Journal of Experimental Psychology: Human Perception \& Performance, 26, 1260-1280.
Lien, M.-C., Schweickert, R., \& Proctor, R. W. (2003). Task switching and response correspondence in the psychological refractory period paradigm. Journal of Experimental Psychology: Human Perception \& Performance, 29, 692-712.

Logan, G. D., \& SCHULKIND, M. D. (2000). Parallel memory retrieval in dual-task situations: I. Semantic memory. Journal of Experimental Psychology: Human Perception \& Performance, 26, 1072-1090.

Mechsner, F., Kerzel, D., KnOblich, G., \& Prinz, W. (2001). Perceptual basis of bimanual coordination. Nature, 414, 69-73.

Pashler, H., \& Baylis, G. (1991). Procedural learning: 2. Intertrial repetition effects in speeded-choice tasks. Journal of Experimental Psychology: Learning, Memory, \& Cognition, 17, 33-48.

Proctor, R. W., \& ReEve, T. G. (1990). Research on stimulus-response compatibility: Toward a comprehensive account. In R. W. Proctor \& T. G. Reeve (Eds.), Stimulus-response compatibility: An integrated perspective (pp. 483-494). Amsterdam: North-Holland.

Teichner, W. H., \& Krebs, M. J. (1974). Laws of visual choice reaction time. Psychological Review, 81, 75-98.

(Manuscript received March 24, 2004; revision accepted for publication November 30, 2004.) 